\title{
EXPERTENSYSTEM FÜR KONFIGURIERUNGSAUFGABEN
}

\author{
Hans-Jürgen Sebastian, RWTH Aachen \\ Karin Müller, ELITE Foundation
}

\begin{abstract}
Konfigurierung ist eine spezielle Form des Designs (des Entwerfens) von komplexen aus elementaren strukturen. Konfigurierungsprobleme treten vor allem in stark strukturierten Domänen, die für Technik und Wirtschaft charakteristisch sind, in vielfältiger Weise und großer Zahl auf. Sie sind in der Regel, bedingt durch die Komponentenstruktur, durch einen großen Lösungsraum charakterisiert. Das Wissen über die zu konfigurierenden systeme und deren komponenten läßt sich in Hierarchien, Relationen und Restriktionen modellieren. Damit bieten sich Konzepte und Techniken aus der Expertensystemtechnologie an, um wissensbasiertes Konfigurieren durchzuführen.

Folgende Methoden kennzeichnen Systeme zur wissenbasierten Konfigurierung:

- eine objektorientierte Repräsentation der Konfigurierungsobjekte,

- die Verwaltung der Relationen und Randbedingungen mit Constraint-Systemen,

- ein Top-Down-Entwurf, der sich an der Komponentenstruktur orientiert.
\end{abstract}

Möchte man in Erweiterung der Möglichkeiten bekannter Konfigurierungs-Expertensysteme auch innovatives Design realisieren, benötigt man eine erweiterte Modellierung des Anforderungsbegriffes. Anforderungen

- an die Funktionalität des zu konfigurierenden Systems,

- in Form von Constraints oder

- als zielkriterien

sind teilweise nur linguistisch sinnvoll formulierbar und können häufig scharf oder unscharf modelliert werden. Beispielsweise kann eine Anforderung wie "gutes Design" sinnvollerweise nur mit Fuzzy-Methoden erfaßt werden. Deshalb haben wir ein Modell zum optimierungsbasierten Konfigurieren unter Einbeziehung unscharfen wissens entwickelt und Kontrollstrategien entworfen und erprobt.

Ein Baustein "Fuzzy-MADM" ist für solche Konfigurierungsaufgaben, bei denen aus einer endlichen Alternativenmenge auszuwählen ist, besonders geeignet. Wir demonstrieren die Funktionalität dieses Moduls "Fuzzy-MADM" innerhalb eines Konfigurierungs-Expertensystemes an einem Beispiel aus der Technik. Die Implementierung ist durch Integration eines Konfigurierungs-Shells mit einem Datenbanksystem, einem 3D-CAD-System und einem Fuzzy-Tool gekennzeichnet. 